Revista de

Contabilidade e

Organizações
DOI: http://dx.doi.org/10.11606/rco.v11i29.125846
Journal of

Accounting and

Organizations

www.rco.usp.br

\title{
Adoção ao padrão IFRS e earnings quality: a persistência do lucro das empresas listadas na BM\&FBovespa
}

\author{
Josimar Pires da Silva ; Mariana Pereira Bonfimª; Jorge Katsumi Niyama ${ }^{\mathrm{a}}$; César Augusto Tibúrcio Silva \\ ${ }^{a}$ Universidade de Brasilia
}

\section{Informações do Artigo}

Histórico do Artigo

Recebido: 28 de janeiro de 2017

Aceito:24 de abril de 2017

Palavras-chave:

Qualidade do Lucro.

Padrão IFRS.

Persistência do Lucro.

\begin{abstract}
Resumo
O objetivo dessa pesquisa foi o de analisar a relação entre a persistência do lucro das empresas listadas na BM\&FBovespa, em comparação ao padrão IFRS. A pesquisa teve como base os dados das demonstrações financeiras, separadas em pré-convergência (anos de 2003 a 2007), e pós-convergência (anos de 2010 a 2014), resultando assim, em uma amostra de 235 empresas, com 2.350 observações. Para o cálculo da proxy de qualidade do lucro, tomou-se por base o modelo presente nos trabalhos de Dechow e Schrand (2004), Dechow, Ge, e Schrand (2010) e Perotti e Wagenhofer (2014), que considera o coeficiente angular $\beta$ como a medida para a persistência nos lucros. Os achados da pesquisa demonstraram que houve aumento na qualidade do lucro após a adoção das IFRS, mostrando consistência com as pesquisas internacionais nas quais a convergência às normas internacionais de contabilidade, poderia resultar em um aumento na qualidade do lucro para as companhias.
\end{abstract}

Copyright (C) 2017 FEA-RP/USP. Todos os direitos reservados

\section{INTRODUÇÃO}

Os lucros de alta qualidade são aqueles que refletem com precisão não só o desempenho atual da empresa, como também são um indicador do desempenho futuro, sendo, por consequência, uma medida do valor da empresa (Dechow \& Schrand, 2004). Os lucros de alta qualidade fornecem informações sobre as características de desempenho de uma empresa que são relevantes para uma decisão dos usuários (Dechow et al. 2010). Em linhas gerais, serão melhores preditores de fluxos futuros de dividendos, e, por conseguinte, melhores preditores do valor da empresa. Dessa forma, as empresas que apresentam lucros de alta qualidade forneceriam informações úteis e necessárias aos usuários da informação contábil, auxiliando-os em sua tomada de decisão.

As pesquisas existentes não têm sido conclusivas se a adoção do padrão IFRS efetivamente melhora a qualidade da informação contábil. Há estudos que apontam que a adoção de um conjunto de padrões contábeis baseados em princípios aumenta o gerenciamento de resultados (Ashbaugh \& Pincus, 2001; Bartov, Goldberg, \& Kim, 2005; Elbannan, 2010; Jeanjean \& Stolowy, 2008). Porém, também há pesquisas que apontam o contrário, ou seja, que a adoção de padrões baseados em princípios aumenta a qualidade da informação contábil, diminuindo o gerenciamento de resultados (Barth, Landsman, \& Lang, 2007; Chen, Tang, Jiang, \& Lin, 2010; Christensen, Lee, Walker, \& Zeng, 2015; Iatridis, 2010; Paananen \& Lin, 2008; Yoon, 2007). As possíveis justificativas consistem em diversos fatores que influenciam nas políticas e práticas contábeis adotadas pelos países, tais como sistema legal, cultura, tipo de mercado de capitais e ambiente social (Askary, 2006).

Segundo Askary (2006), os valores compartilhados dentro das fronteiras nacionais têm o potencial de promover e sustentar diferenças significativas no comportamento social, tais como as práticas contábeis. As diferenças de práticas contábeis refletem manifestações da vida social nacional de diferentes sistemas. Em virtude disso, pode-se afirmar que a cultura e a contabilidade são indissociáveis. A contabilidade brasileira, esta, em princípio, possui características do modelo continental, (Niyama, 2010; Nobes \& Parker, 2010), assim como são

Autor Correspondente: Tel (61) 3107-0254

E-mail: josimarnx@yahoo.com.br (J. P. Silva); marianapbonfim@gmail.com (M. P. Bonfim); jorgekatsumi@gmail.com (J. K. Niyama);

cesaraugustotiburciosilva@gmail.com (C. A. T. Silva).

Universidade de Brasília, Campus Universitário Darcy Ribeiro, Asa Norte, 70.910-900, Brasília - DF, Brasil. 
os casos da França, Alemanha, Itália, Japão, Bélgica, Espanha, países comunistas (Europa Oriental), países da América do Sul, entre outros. Este grupo de países possuI as seguintes características predominantes: a) profissão contábil fraca e pouco atuante; b) forte interferência governamental no estabelecimento de padrões contábeis, notadamente aqueles de natureza fiscal; c) demonstrações financeiras que visam atender primeiramente aos credores e ao governo, ao invés dos investidores e; d) importância de bancos e outras instituições financeiras, inclusive governamentais, como fonte de captação pelas empresas, em detrimento dos recursos provenientes do mercado de acionário.

No entanto, a adoção do padrão contábil IFRS pelo Brasil que teve como marco a criação do Comitê de Pronunciamentos Contábeis (CPC), poderia ser considerado um afastamento do modelo continental e uma aproximação do modelo anglo-saxão, cujas características, segundo Niyama (2010), são: existência de uma profissão contábil forte e atuante; sólido mercado de capitais, como fonte de captação de recursos; pouca interferência governamental na definição de normas; e investidores atuando como principais usuários. Esses fatores, sobretudo uma profissão contábil forte, definindo padrões contábeis, podem resultar na produção de um arcabouço teórico que possibilite a emissão de relatórios financeiros que representem a essência econômica das transações. Em vista desses fatos, é esperado que o padrão IFRS resulte em maior qualidade da informação contábil.

Com base no contexto previamente destacado, e nas razões elencadas, o problema de pesquisa pode ser descrito através da seguinte pergunta: como a adoção ao padrão IFRS influencia a qualidade do lucro das empresas brasileiras? Em decorrência deste problema apresentado, esta investigação tem como objetivo verificar a qualidade do lucro com a adoção das normas internacionais de contabilidade. A principal diferença dessa pesquisa em relação aos estudos anteriores, e, portanto, a sua principal contribuição, é a comparação entre os períodos anteriores e posteriores a convergência às normas internacionais de contabilidade no Brasil, sob a ótica da qualidade do lucro. As pesquisas anteriores, como a de Pimentel e Aguiar (2012) e a de Santos e Cavalcante (2014), também analisaram a relação entre qualidade do lucro e adoção ao padrão IFRS, mas não fizeram o contraponto entre os dois períodos.

\section{QUALIDADE DO LUCRO E ADOÇÃO AO PADRÃO IFRS}

Hail, Leuz e Wysocki (2010a, 2010b) fizeram uma revisão das pesquisas e constataram que os benefícios da convergência dependem da empresa, do setor de atuação, dos mercados e dos países que optaram pelas normas IFRS. Os autores destacam que diversos fatores podem influenciar a prática contábil e que a adoção das IFRS não garante benefícios para um determinado país. A adoção das IFRS pelos Estados Unidos, por exemplo, poderia ter efeito reduzido ou até negativo, segundo os autores.

Pesquisas anteriores sugerem que empresas que adotam IFRS geralmente apresentam maior qualidade da contabilidade do que aquelas que utilizam GAAPs locais (por exemplo, Barth et al., 2007). Além disso, de acordo com Barth, Landsman, Lang e Williams (2012), após a adoção do padrão IFRS, pôde-se verificar que um aumento na qualidade, melhora também a comparabilidade dos valores contábeis. Os benefícios ao mercado de capitais com a adoção do padrão IFRS incluem a redução do custo de capital, melhoria da liquidez (Daske, Hail, Leuz \& Verdi, 2008; Li, 2010), aumento do investimento estrangeiro (Covrig, Defond \& Hung, 2007) e redução na dispersão de previsões de analistas (Byard et al., 2010; Horton, Serafeim, \& Serafeim, 2013; Tan, Wang, \& Welker, 2011). Coletivamente, estes estudos sugerem ainda que firmas que aplicam IFRS apresentam maior qualidade nos relatórios financeiros e uma maior comparabilidade do que aquelas que aplicam normas contábeis nacionais (Landsman, Maydew \& Thornock, 2012).

A implementação das IFRS possibilita a redução da assimetria de informação entre investidores informados e desinformados (Bushman \& Smith, 2001) além de reduzir o nível de incerteza dos investidores sobre o desempenho futuro da empresa, proporcionando então menor custo de capital (Samarasekera, 2013). A redução da incerteza e da assimetria de informações facilitaria a comunicação entre os gestores e outras partes interessadas relacionadas, tais como acionistas, credores, autoridades reguladoras e de supervisão e analistas financeiros (Iatridis, 2010). Isso tenderia a reduzir os custos de agência que possam surgir (Bushman \& Smith, 2001; Healy \& Palepu, 2001), e por sua vez, conduzir a uma elevação nos retornos das ações, o que poderia estar relacionado ao desempenho financeiro atual da empresa (Gelb \& Zarowin, 2002).

Adicionalmente, a redução da assimetria da informação leva a custos mais baixos na emissão de capital próprio (Leuz \& Verrecchia, 2000), e da dívida (Sengupta, 1998) facilitando a obtenção de financiamentos via 
capital próprio e de terceiros (El-Gazzar, Finn \& Jacob, 1999). Tal redução também ajuda os investidores a comparar o valor de investimentos alternativos, o que promove a divulgação e comparabilidade dos relatórios financeiros e também auxilia os investidores e credores a monitorar o desempenho de insiders (Samarasekera, 2013). Sendo assim, os benefícios da implementação das IFRS incluem a harmonização das práticas contábeis em todos os países que as adotam por sua vez, isso leva a uma maior comparabilidade, menores custos de transação e a melhora nos investimentos internacionais. As normas internacionais também auxiliam os investidores na tomada de decisões e previsões acerca do desempenho futuro da firma (Niyama, 2010; Nobes \& Parker, 2010).

Essencialmente, a adoção das IFRS fornece um sinal positivo da contabilidade em direção a uma maior qualidade e transparência (Tendeloo \& Vanstraelen, 2005). Barth et al. (2007), Chen et al. (2010) e Iatridis (2010) sustentam que a maior qualidade das normas e os elevados requisitos de evidenciação do IASB propiciam aumento na qualidade das informações contábeis divulgadas principalmente em países com mecanismos de proteção ao investidor e mercado acionário forte, levando à redução nos níveis de gerenciamento de resultado. Na mesma linha, Daske e Gebhardt (2006) relatam que firmas que implementam IFRS voluntária ou obrigatoriamente, tendem a apresentar maior qualidade da divulgação do que firmas que usam as normas nacionais. O fornecimento de divulgações contábeis de qualidade tenderia a reduzir as possibilidades de manipulação de resultados e aumentar a eficiência (Baiman \& Verrecchia, 1996; Kasznik, 1999; Leuz, 2003). Os requisitos de maiores divulgações e elevada qualidade da informação financeira que resultam do padrão IFRS, implicam que a adoção dessas normas sinaliza de forma positiva aos investidores, por meio de menores custos de assimetria de informação e agência (Tarca, 2004).

Entretanto, é importante ressaltar que muitas conclusões sobre as vantagens da implantação das IFRS são resultantes de pesquisas realizadas em países onde a qualidade da informação antes da implantação era menor, como provavelmente ocorreu com o Brasil. Em países onde as normas nacionais são de boa qualidade, como é o caso dos Estados Unidos, talvez estas vantagens não sejam evidentes ou até não existam. Outro aspecto relevante refere-se ao custo da adoção das normas internacionais: estes custos envolvem treinamento, mudança de softwares, regulamentações, etc. Podem também incluir o aumento da presença das grandes empresas de auditoria no mercado interno. Estes aspectos geralmente não foram considerados como foco central das pesquisas citadas anteriormente, muito embora sejam relevantes para uma discussão (Niyama \& Silva, 2013).

Um aspecto desta discussão diz respeito aos lucros de qualidade mais elevados a partir da adoção das IFRS. Os lucros de qualidade fornecem informações sobre as características de desempenho financeiro de uma empresa que são relevantes para uma decisão específica escolhida por um tomador de decisão (Dechow et al., 2010). A qualidade dos lucros geralmente é destacada a partir da perspectiva dos analistas (Dechow \& Schrand, 2004), uma vez que é relevante para a precificação de títulos negociados publicamente. Essa ainda é uma das questões mais importantes e ainda controversas na pesquisa contábil recente. Segundo Dechow e Schrand (2004) lucro de alta qualidade significa que os números refletem com precisão o valor intrínseco da empresa. Tais lucros são referidos na literatura contábil como permanentes (Beaver, 1998; Black, 1980; Ohlson \& Zhang, 1998). Outra maneira de pensar sobre este conceito é que os lucros são de alta qualidade quando o retorno sobre o capital próprio é uma boa medida da taxa interna de retorno do portfólio atual da empresa (Dechow \& Schrand, 2004).

Diversas pesquisas têm evidenciado a relação entre qualidade do lucro e adoção do padrão IFRS. No entanto os resultados nem sempre são convergentes: alguns estudos apontam que a adoção do padrão IFRS resulta em maior qualidade da informação contábil (Ashbaugh \& Pincus, 2001; Barth et al., 2007; Chen et al., 2010; Christensen et al., 2015; Iatridis, 2010; Paananen \& Lin, 2008; Psaros \& Trotman, 2004; Yoon, 2007) ao passo que outros, em menor qualidade (Bartov et al., 2005; Elbannan, 2010; Jeanjean \& Stolowy, 2008; Kajimoto \& Nakao, 2015; Klann \& Beuren, 2012).

As pesquisas já realizadas sobre a adoção às normas internacionais de contabilidade e qualidade do lucro no Brasil, em geral, não são conclusivas ou não são observadas diferenças na qualidade do lucro das empresas pesquisadas (Santos, Lima, Freitas, \& Lima, 2011; Sousa, Sousa, \& Demonier, 2016). Além disso, não foram encontradas pesquisas empíricas no mercado brasileiro sobre o assunto com o mesmo horizonte temporal, ou seja, realizando uma comparação entre o período anterior e o período posterior à convergência. Essa demarcação de período e a consideração de que o período pós-convergência abrange o full IFRS podem ser consideradas como a principal diferença dessa pesquisa para as demais.A partir dessas evidências, concernentes à qualidade do lucro e à adoção das International Financial Reporting Standards (padrão IFRS), foi possível elaborar a seguinte hipótese de pesquisa:

$\mathrm{H}_{1}=$ existe uma relação significante e positiva entre a adoção ao padrão IFRS e Qualidade do Lucro das empresas 


\section{PROCEDIMENTOS METODOLÓGICOS}

Nesta pesquisa foram utilizados os dados das demonstrações financeiras publicadas e disponibilizadas ao público em geral no sítio eletrônico da BM\&FBovespa e extraídos através do software de divulgação externa EmpresasNet. Inicialmente, a amostra compreende 494 empresas dos 10 setores da BM\&FBovespa (bens industriais; construção e transporte; consumo cíclico; consumo não cíclico; materiais básicos; petróleo; tecnologia da informação; telecomunicações; utilidade pública; e financeiros) abrangendo um período de 10 períodos/anos separados em pré-convergência (2003 a 2007) e pós-convergência (2010 a 2014). Não serão analisados os anos de 2008 e 2009, em decorrência da proximidade com o início do processo de convergência no Brasil.

A amostra final resultou em 2.350 observações (1.175 para o período pré-convergência e 1.175 para o período pós-convergência) de 235 empresas, visto que 259 companhias foram excluídas da amostra, pois não dispunham da totalidade dos dados necessários para a realização da pesquisa. No que tange à proxy de qualidade do lucro, a presente pesquisa toma por base os trabalhos de Dechow e Schrand (2004), Dechow, Ge e Schrand (2010), e foi utilizada a persistência dos lucros, frequentemente abordada na literatura.

A base para mensuração do lucro é o lucro líquido (LL). A métrica utilizada, a Persistência, é igual ao coeficiente angular da seguinte regressão:

$$
\mathrm{LL}_{\mathrm{i}, \mathrm{t}}=\alpha+\beta \mathrm{LL}_{\mathrm{i}, \mathrm{t}-\mathrm{l}}+\varepsilon_{\mathrm{i}, \mathrm{t}}
$$

Em que:

$\mathrm{LL}_{\mathrm{i}, \mathrm{t}}=$ lucro líquido do período $\mathrm{t}$, dividido pelo ativo total no início do período $\mathrm{t}$.

$\mathrm{LL}_{\mathrm{i}, \mathrm{t}-1}=$ lucro líquido do período $\mathrm{t}-1$, dividido pelo ativo total no início do período $\mathrm{t}-1$.

A utilização da persistência se justifica visto que se encaixa com a visão de que os lucros funcionam como uma métrica sumária de fluxos de caixa esperados, úteis, para a avaliação dos títulos (Dechow et al., 2010) tendo por premissa que as informações contábeis históricas têm valor preditivo (Olson \& Mossman, 2003) e os valores das empresas são indicados por informação nas demonstrações financeiras, sobretudo o lucro (Ou \& Penman, 1989). Desta maneira, as empresas com lucros mais persistentes têm lucros/fluxo de caixa mais sustentáveis que irão torná-los mais úteis na avaliação dos preços das ações (Dechow et al., 2010; Dechow \& Schrand, 2004; Perotti \& Wagenhofer, 2014). A proxy de qualidade do lucro é apresentada pelo $\beta$ da equação de regressão (1) com o propósito de observar o comportamento das empresas listadas na BM\&FBovespa: se no período pré-convergência (2003 a 2007) a qualidade do lucro foi menor do que no período pós-convergência (2010 a 2014).

\section{ANÁLISE DOS RESULTADOS}

A estatística descritiva dos dados das 235 companhias listadas na BM\&FBovespa estão evidenciadas na tabela 1. Por meio da análise dos dados desta tabela, no que diz respeito ao desvio padrão, o período pósconvergência (2010 a 2014) apresentou um valor menor do que o período pré-convergência (2003 a 2007); isso indica que a adoção ao padrão IFRS levou a uma menor variabilidade do lucro líquido das empresas listadas na BM\&FBovespa. Com isso, pode-se considerar que a redução corrobora a concepção de que o lucro das companhias, após a adoção das normas internacionais de contabilidade, se tornou mais informacional, ou seja, uma contabilidade baseada em princípios aumenta a qualidade do lucro das empresas. 
Tabela 1. Estatística descritiva da amostra

\begin{tabular}{ccccccc}
\hline & \multicolumn{2}{c}{$\begin{array}{c}\text { Período pré-convergência } \\
\text { (2003 a 2007) }\end{array}$} & \multicolumn{2}{c}{$\begin{array}{c}\text { Período pós-convergência } \\
\text { (2010 a 2014) }\end{array}$} \\
& Média & Mediana & $\begin{array}{c}\text { Desvio } \\
\text { padrão }\end{array}$ & Média & Mediana & $\begin{array}{c}\text { Desvio } \\
\text { padrão }\end{array}$ \\
\hline LL & 0,0445 & 0,0403 & 0,1782 & 0,0180 & 0,0268 & 0,1326 \\
\hline Fonte: Elaborada pelos autores.
\end{tabular}

Fonte: Elaborada pelos autores.

Quanto a informatividade do lucro, existem evidências de que o lucro suavizado é mais informacional para as empresas de alta qualidade do lucro (Bao \& Bao, 2004), apresentando melhor performance tanto para as empresas de alta quanto de baixa qualidade do lucro (Baseri, Ranjbar, \& Khademi, 2013). A mudança no preço atual das ações de empresas com maior suavização de resultados contém mais informações sobre os seus ganhos futuros do que a mudança no preço das ações de empresas com menores suavizações (Tucker \& Zarowin, 2006) e maior persistência (Silhan, 2014).

Com o intuito de analisar a normalidade dos resíduos, realizou-se o teste de Jarque-Bera, que exibiu um resultado de 0,00 , não rejeitando assim a hipótese nula, ou seja, atestando a normalidade dos resíduos da regressão. Com relação ao pressuposto da homocedasticidade dos resíduos, foi realizado o teste de White, que tem como hipótese nula a não existência de heterocedasticidade. Com uma probabilidade de 0,9991 e uma distribuição quiquadrado com 2 graus de liberdade, não se rejeitou a hipótese nula e atestou-se a homocedasticidade dos erros.

Para analisar a autocorrelação dos resíduos da regressão, foi utilizado o teste de Durbin-Watson (DW), que tem como hipótese nula a ausência de autocorrelação. O resultado do teste DW mostrou um valor de 2,221, estando assim, inserido na área de não rejeição da hipótese nula do teste, de modo que foi verificado que os resíduos não são autocorrelacionados.

Foi realizado também o teste de Breusch-Pagan-Godfrey que corroborou a homocedasticidade dos resíduos da regressão $(0,9985)$, indicando assim, que não é possível utilizar o modelo de efeitos aleatórios. Dessa forma, foi utilizado o teste de Chow e os critérios de Akaike, Schwarz e Hannan-Quinn para verificar qual modelo regressivo é o mais ajustado: efeito agrupado (pooled) ou efeitos fixos. Como pelo modelo de regressão agrupada (pooled) foram obtidos os menores valores dos critérios mencionados acima, foi verificado que esse é o modelo mais ajustado. Adotando-se então a abordagem pooled, os resultados das regressões (pré e pós-convergência) podem ser visualizados na Tabela 2.

Tabela 2. Resultados da estimação da regressão em painel pelo método pooled - variável dependente

\begin{tabular}{|c|c|c|c|c|c|c|}
\hline \multirow[t]{2}{*}{ Variáveis } & \multicolumn{3}{|c|}{$\begin{array}{c}\text { Período pré-convergência } \\
\text { (2003-2007) }\end{array}$} & \multicolumn{3}{|c|}{$\begin{array}{c}\text { Período pós-convergência } \\
(2010-2014)\end{array}$} \\
\hline & Coeficiente & Erro Padrão & p-valor & Coeficiente & Erro Padrão & p-valor \\
\hline \multirow[t]{2}{*}{ Constante } & 0,097979 & 0,006949 & $0,0000^{*}$ & $-0,004977$ & 0,002654 & 0,0610 \\
\hline & 0,227343 & 0,017095 & $0,0000^{*}$ & 0,359895 & 0,020001 & $0,0000 *$ \\
\hline $\mathrm{R}^{2}$ & 0,131023 & & & 0,216323 & & \\
\hline $\mathrm{R}^{2}$ ajustado & 0,130282 & & & 0,215655 & & \\
\hline Estatística F & 176,8631 & & & 323,7899 & & \\
\hline p-valor (Est. F) & 0,000000 & & & 0,000000 & & \\
\hline Durbin-Watson & 1,596358 & & & 1,740044 & & \\
\hline
\end{tabular}

Fonte: Elaborada pelos autores.

Obs.: * indica a significância ao nível de $1 \%$.

Os dados da regressão evidenciaram que a variável apresentou coeficientes significantes a 1\% (p-valor $=0,000)$ e positivo para com a variável dependente, corroborando assim, a hipótese de pesquisa de que existe uma relação significante e positiva entre a adoção ao padrão IFRS e a qualidade do lucro das empresas listadas na BM\&FBovespa. A base para a mensuração da persistência do lucro líquido é o coeficiente angular $\beta$ da regressão (1), em que índices maiores indicam uma maior persistência do lucro, ou seja, os lucros são mais informacionais; e índices menores, indicam uma menor qualidade do lucro. 
Conforme observado na Tabela 2 , o período pré-convergência às IFRS apresentou um $\beta$ de 0,227 enquanto o período pós-convergência, apresentou um coeficiente de 0,359 , indicando assim, um aumento no $\beta$ da regressão, ao comparar o período antes da adoção com o período após a adoção das IFRS. Esse resultado corrobora a hipótese de pesquisa, indicando que a adoção às IFRS, pelas empresas listadas na BM\&FBovespa, aumentou a qualidade do lucro dessas companhias. Adicionalmente, foram realizadas regressões dividindo os setores da BM\&FBovespa, a fim de verificar se a adoção às IFRS influencia na qualidade do lucro das empresas. Para isso, foram criadas dummys para cada setor da BM\&FBovespa e foi estimada a seguinte equação de regressão:

$$
\begin{aligned}
& \mathrm{LL}_{\mathrm{i}, \mathrm{t}}=\alpha+\beta_{1}\left(\mathrm{BI} * \mathrm{LL}_{\mathrm{i}, \mathrm{t}-1}\right)+\beta_{2}\left(\mathrm{CT} * \mathrm{LL}_{\mathrm{i}, \mathrm{t}-1}\right)+\beta_{3}\left(\mathrm{CC} * \mathrm{LL}_{\mathrm{i}, \mathrm{t}-1}\right)+\beta_{4}\left(\mathrm{CNC} * \mathrm{LL}_{\mathrm{i}, \mathrm{t}-1}+\beta_{5}\left(\mathrm{FIN} * \mathrm{LL}_{\mathrm{i}, \mathrm{t}-1}\right)+\right. \\
& \beta_{6}\left(\mathrm{MB} * \mathrm{LL}_{\mathrm{i}, \mathrm{t}-1}\right)+\beta_{7}\left(\mathrm{PGB} * \mathrm{LL}_{\mathrm{i}, \mathrm{t}-1}\right)+\beta_{8}\left(\mathrm{TI} * \mathrm{LL}_{\mathrm{i}, \mathrm{t}-1}\right)+\beta_{9}\left(\mathrm{TEL} * \mathrm{LL}_{\mathrm{i}, \mathrm{t}-1}\right)+\beta_{10}\left(\mathrm{UP} * \mathrm{LL}_{\mathrm{i}, \mathrm{t}-1}\right)+\varepsilon_{\mathrm{i}, \mathrm{t}}
\end{aligned}
$$

As variáveis BI, CT, CC, CNC, FIN, MB, PGB, TI, TEL e UP são representativas dos setores da BM\&FBovespa (bens industriais; construção e transporte; consumo cíclico; consumo não cíclico; materiais básicos; petróleo; tecnologia da informação; telecomunicações; utilidade pública; e, financeiros, respectivamente) e binárias, sendo igual a 1 quando a empresa está inserida no respectivo setor, e 0 caso contrário. Os resultados obtidos a partir da regressão (2) podem ser vistos na Tabela 3, abaixo:

\begin{tabular}{|c|c|c|c|c|c|c|}
\hline \multirow[t]{2}{*}{ Variáveis } & \multicolumn{3}{|c|}{$\begin{array}{l}\text { Período pré-convergência } \\
\qquad(2003-2007)\end{array}$} & \multicolumn{3}{|c|}{$\begin{array}{l}\text { Período pós-convergência } \\
\qquad(2010-2014)\end{array}$} \\
\hline & Coeficiente & Erro Padrão & p-valor & Coeficiente & Erro Padrão & p-valor \\
\hline Constante & 0,075096 & 0,005161 & $0,0000^{*}$ & $-0,001223$ & 0,004100 & 0,7655 \\
\hline$B I^{*}$ & 0,303606 & 0,017351 & $0,0000 *$ & 0,746880 & 0,089179 & $0,0000^{*}$ \\
\hline$C T^{*}$ & 0,288144 & 0,114422 & $0,0119 *$ & 0,503967 & 0,063080 & $0,0000^{*}$ \\
\hline$C C^{*}$ & 0,476537 & 0,164082 & $0,0038^{*}$ & 0,400585 & 0,086306 & $0,0000^{*}$ \\
\hline$C N C^{*}$ & $-0,076701$ & 0,155703 & 0,6224 & 0,168354 & 0,070166 & $0,0166^{*}$ \\
\hline$F I N^{*}$ & 0,518504 & 0,056737 & $0,0000 *$ & 0,307430 & 0,058485 & $0,0000^{*}$ \\
\hline$M B^{*}$ & 0,495804 & 0,086422 & $0,0000 *$ & 0,137746 & 0,086168 & 0,1102 \\
\hline$P G B^{*}$ & 0,481019 & 0,053157 & $0,0000 *$ & 0,646743 & 0,092553 & $0,0000^{*}$ \\
\hline$T I^{*}$ & 0,231791 & 0,010153 & $0,0000 *$ & 0,304135 & 0,138957 & $0,0288^{*}$ \\
\hline$T E L *$ & 1,533453 & 0,261051 & $0,0000 *$ & 0,733822 & 0,060734 & $0,0000^{*}$ \\
\hline$U P^{*}$ & 0,033727 & 0,010578 & $0,0015^{*}$ & 0,250741 & 0,087698 & $0,0043 *$ \\
\hline $\mathrm{R}^{2}$ & 0,319474 & & & 0,263552 & & \\
\hline $\mathrm{R}^{2}$ ajustado & 0,313628 & & & 0,257225 & & \\
\hline Estatística F & 54,64415 & & & 41,65600 & & \\
\hline p-valor (Est. F) & 0,000000 & & & 0,000000 & & \\
\hline Durbin-Watson & 2,011927 & & & 1,933601 & & \\
\hline
\end{tabular}

Tabela 3. Resultados da estimação da regressão pelos setores da BM\&FBovespa (pooled) - variável dependente

Fonte: Elaborada pelos autores.

Obs.: * indica a significância ao nível de $1 \%$.

Analisando a Tabela 3, pode-se perceber que, dos 10 setores em que são divididas as companhias na BM\&FBovespa, 6 deles apresentaram aumento no coeficiente angular $\beta$ após a adoção ao padrão IFRS. Os setores de bens industriais, construção e transporte, consumo não cíclico, petróleo, gás e combustível, tecnologia da informação e de utilidade pública exibiram aumento na qualidade do lucro após a implementação das normas internacionais de contabilidade, o que denota não só a maioria dos setores (6 no total), como também a maior parte das empresas pesquisadas (122 ou 52\%).

Os setores de bens industriais, construção e transporte, petróleo, gás e combustíveis são caracterizados por altos investimentos em imobilizados; após o advento da convergência contábil, as empresas listadas na BM\&FBovespa passaram a adotar taxas de depreciação diferentes das taxas de depreciação fiscal. Pesquisas como 
a de Eckert et al. (2012) constataram que após a introdução das normas internacionais de contabilidade no Brasil, foi possível perceber alterações na forma de avaliação da vida útil dos imobilizados e reduções das despesas de depreciação. A exemplo, o setor de bens industriais se trata essencialmente de indústrias que produzem bens de capital e necessitam, portanto, de altos investimentos em ativos imobilizados (Eckert et al., 2012; Freire, Machado, Machado, Souza \& Oliveira, 2012), implicando em maiores valores depreciados. A expectativa é que o tratamento contábil das depreciações nesse setor seja mais acurado, devido à importância que esses valores representam nos ativos e resultados das empresas pesquisadas (Freire et al., 2012). No geral, a adoção do padrão IFRS, sobretudo em relação à avaliação dos imobilizados nestes setores, possibilita melhor qualidade da informação contábil em virtude da depreciação com base na vida útil econômica e do teste de recuperabilidade de ativos.

O setor de consumo não-cíclico comporta as empresas que se dedicam à venda de bens alimentares, bebidas, tabaco e produtos de casa. Este setor caracteriza-se pela sua menor correlação com a evolução da economia: em épocas de menor crescimento econômico, ou mesmo de recessão, o consumo de produtos tende a não ser particularmente afetado. Os bens essenciais de consumo têm uma menor elasticidade no preço do que quaisquer outros produtos e serviços. Significa isto que quando os preços aumentam, a procura por produtos não se ressente tanto quanto a procura por outros gêneros de produtos, como por exemplo, os tecnológicos (Horta, Alves, \& Jorge, 2013).

Adicionalmente, o setor das empresas petrolíferas com grande relevância no Produto Interno Bruto (PIB) e na economia nacional, possui, grandes negócios transacionados através do petróleo, envolvendo elevado volume de operações financeiras e, consequentemente, de diversas transações contábeis exigidas para atender à demanda informacional. Não obstante, o setor também atua em ambiente de comércio exterior, o que naturalmente pode levar a uma necessidade de transmitir suas informações em nível igualitário e padronizado (Ribeiro \& Da Silva, 2010).

Por outro lado, 4 setores apresentaram menores coeficientes após a adoção das IFRS: consumo cíclico, financeiro, materiais básicos e telecomunicações. Possivelmente, esses resultados foram obtidos porque o setor financeiro e o setor de telecomunicações ainda possuem forte regulação no Brasil: ainda que seus órgãos reguladores tenham adotado alguns pronunciamentos do CPC, essas companhias ainda estão sujeitas a uma forte regulação por parte desses órgãos e a sua contabilidade ainda não é totalmente baseada em princípios. Já no setor de materiais básicos, 70\% das companhias pertencem aos subsetores de mineração, químicos e siderurgia e metalurgia, também marcados por uma forte regulação, o que pode ter influenciado na qualidade do lucro dessas companhias.

Os resultados encontrados nessa pesquisa vão ao encontro da hipótese pesquisada de que a adoção ao padrão IFRS aumenta a qualidade do lucro das companhias, tornando-o melhor preditor dos fluxos futuros de dividendos e do valor das ações dessas companhias. Desse modo, os achados vão de encontro aos trabalhos de Psaros e Trotman (2004), Ashbaugh e Pincus (2001), Yoon (2007), Barth, Landsman e Lang (2008), Christensen et al. (2015), Paananen e Lin (2009), Chen et al. (2010) e Iatridis (2010) nos quais foi evidenciado que a adoção às normas internacionais de contabilidade resulta em lucros de maior qualidade.

\section{CONSIDERAÇÕES FINAIS}

Os resultados corroboraram a hipótese de pesquisa indicando que existe uma relação significante e positiva entre a adoção ao padrão IFRS e a qualidade do lucro das empresas listadas na BM\&FBovespa. Além disso, foi utilizado o coeficiente angular $\beta$ da regressão (1) como forma de medir a persistência dos lucros: como o período pós-convergência apresentou um coeficiente maior do que no período pré-convergência, pôde-se constatar que a adoção ao padrão IFRS aumentou a qualidade do lucro dessas companhias. Esse resultado foi consistente com os trabalhos de Psaros e Trotman (2004), Ashbaugh e Pincus (2001), Yoon (2007), Barth, Landsman e Lang (2008) que evidenciaram que a implementação das normas internacionais leva as companhias a um aumento na qualidade de seus lucros, tornando-o uma medida de interesse para os investidores, uma vez que ele se torna um melhor preditor do valor das ações dessas companhias.

Adicionalmente, foi realizada a regressão (2) com a inserção de variáveis dummys com o intuito de separar os 10 setores da BM\&FBovespa e verificar a relação entre a adoção ao padrão IFRS e qualidade do lucro, por setor. Foi verificado, portanto, que a maioria dos setores e das companhias exibiram aumento na qualidade do lucro ao implementarem as normas internacionais de contabilidade. Como sugestão para pesquisas futuras, indica-se o estudo de outras abordagens de mensuração da qualidade do lucro, como a magnitude dos accruals ou 
a suavização, como os apresentados no trabalho de Dechow, Ge e Schrand (2010), por exemplo.

\section{REFERÊNCIAS}

Ashbaugh, H., \& Pincus, M. (2001). Domestic Accounting Standards , International Accounting Standards , and the Predictability of Earnings, 39(3), 417-434.

Askary, S. (2006). Accounting professionalism - a cultural perspective of developing countries. Managerial Auditing Journal, 21(1), 102-111.

Baiman, S., \& Verrecchia, R. E. (1996). The Relation among Capital Markets, Financial Disclosure, Production Efficiency, and Insider Trading. Journal of Accounting Research, 34(1), 1-22.

Bao, B. H., \& Bao, D. H. (2004). Income smoothing, earnings quality and firm valuation. Journal of Business Finance and Accounting, 31(9-10), 1525-1557.

Barth, M. E., Landsman, W. R., \& Lang, M. H. (2007). International Accouting Standards and Accouting Quality. Journal of Accounting Research.

Barth, M. E., Landsman, W. R., Lang, M., \& Williams, C. (2012). Are IFRS-based and US GAAP-based accounting amounts comparable? Journal of Accounting and Economics, 54(1), 68-93.

Bartov, E., Goldberg, S. R., \& Kim, M. (2005). Comparative Value Relevance Among German, U.S., and International Accounting Standards: A German Stock Market Perspective. Journal of Accounting, Auditing \& Finance, 20(2), 95-119.

Baseri, M., Ranjbar, A., \& Khademi, S. (2013). Comparative Investigarion of Income Smoothing and Earning Quality on Firm Valuation of Listed Companies in Tehran Stock Exchange. Advances in Environmental Biology, 7(10), 2992.

Beaver, W. H. (1998). Financial Reporting: An Accounting Revolution. (P. Hall., Ed.) (3rd ed.). Englewood Cliffs, NJ.

Black, F. (1980). The Mlagic in Earnings : Ecunumic Earnings versus Earnings Accsu . nhing. Financial Analysts Journal, 36(6), 19-24.

Bushman, R. M., \& Smith, A. J. (2001). Financial Accounting Information and Corporate Governance. Journal of Accounting \& Economics, 23, 1-115.

Byard, D., Brown, A., Dahya, J., Darrough, M., Davis-friday, P., \& Gore, A. (2010). The Effect of Mandatory IFRS Adoption on Financial Analysts 'Information Environment. Journal of Accounting Research, 49, 69-96.

Chen, H., Tang, Q., Jiang, Y., \& Lin, Z. (2010). The Role of International Financial Reporting Standards in Accounting Quality: Evidence from the European Union. Journal of International Financial Management Accounting, 21(3), 220-278.

Christensen, H. B., Lee, E., Walker, M., \& Zeng, C. (2015). Incentives or Standards: What Determines Accounting Quality Changes around IFRS Adoption? European Accounting Review, 24(1), 31-61.

Covrig, V. M., Defond, M. L., \& Hung, M. (2007). Home bias, foreign mutual fund holdings, and the voluntary adoption of international accounting standards. Journal of Accounting Research, 45(1), 41-70.

Daske, H., \& Gebhardt, G. (2006). International financial reporting standards and experts' perceptions of disclosure quality. Abacus, 42(3-4), 461-498. http://doi.org/10.1111/j.1467-6281.2006.00211.x

Daske, H., Hail, L., Leuz, C., \& Verdi, R. (2008). Mandatory IFRS Reporting around theWorld: Early Evidence on the Economic Consequences. Journal of Accounting Research, 46(5), 1085-1142.

Dechow, P., Ge, W., \& Schrand, C. (2010). Understanding earnings quality: A review of the proxies, their determinants and their consequences. Journal of Accounting and Economics, 50(2-3), 344-401. http://doi. org/10.1016/j.jacceco.2010.09.001

Dechow, P. M., \& Schrand, C. M. (2004). Earnings quality. Research Foundation of CFA Institute, 1-160. Retrieved 
from http://scholar.google.com/scholar?hl=en\&btnG=Search\&q=intitle:Earnings+quality\#2

Eckert, A., Mecca, M. S., Biasio, R., \& Souza, K. M. de. (2012). Normas contábeis de reconhecimento e mensuração do ativo imobilizado: impacto em empresas de bens industruais gauchas listadas na BM\&FBOVESPA. Revista Contabilidade E Controladoria, 4(2), 69-89.

El-Gazzar, S., Finn, P. M., \& Jacob, R. (1999). An Empirical Investigation of Multinational Firms' Compliance with International Accounting Standards. The International Journal of Accounting, 34(2), 239-248.

Elbannan, M. A. (2010). Accounting and stock market effects of international accounting standards adoption in an emerging economy. Review of Quantitative Finance and Accounting, 36(2), 207-245.

Freire, M. D. D. M., Machado, M. R. R., Machado, L. S., Souza, E. S., \& Oliveira, J. J. De. (2012). Aderência às normas internacionais de contabilidade pelas empresas brasileiras. Revista de Contabilidade E Organizações, $6(15), 3-22$.

Gelb, D. S., \& Zarowin, P. (2002). Corporate disclosure policy and the informativeness of stock prices. Review of Accounting Studies, 7(1), 33-52.

Hail, L., Leuz, C., \& Wysocki, P. (2010a). Global Accounting Convergence and the Potential Adoption of IFRS by the U.S. (Part II): Political factors and future scenarios for U.S. accounting standards. Accounting Horizons, 24(4), 567-588.

Hail, L., Leuz, C., \& Wysocki, P. (2010b). Global Accounting Convergence and the Potential Adoption of IFRS by the U.S. (Parte I): Conceptual Underpinnings and Economic Analysis. Accounting Horizons, 24(3), 355-394.

Healy, P. M. ., \& Palepu, K. G. (2001). A Review of the Empirical Disclosure Literature. Journal of Chemical Information and Modeling, 53(December), 160.

Horta, R. A. M. H., Alves, F. J. dos S., \& Jorge, M. J. (2013). Descontinuidade de Empresas Brasileiras do Setor de Consumo Não Cíclico : Um Estudo Com Dados Contábeis Utilizando Técnicas de Data Mining. Revista de Informação Contábil, 7(3), 63-83.

Horton, J., Serafeim, G., \& Serafeim, I. (2013). Does mandatory IFRS adoption improve information comparability? Contemporary Accounting Research, 30(1), 388-423.

Iatridis, G. (2010). International Financial Reporting Standards and the quality of financial statement information. International Review of Financial Analysis, 19(3), 193-204.

Jeanjean, T., \& Stolowy, H. (2008). Do accounting standards matter? An exploratory analysis of earnings management before and after IFRS adoption. Journal of Accounting and Public Policy, 27(6), 480-494.

Kajimoto, C. G. K., \& Nakao, S. H. (2015). Persistência dos Lucros Tributáveis com a Adoção das IFRS no Brasil. XII Congresso USP Iniciação Científica Em Contabilidade, 1-16.

Kasznik, R. (1999). On the Association between Voluntary Disclosure and Earnings Management. Journal of Accounting Research, 37(1), 57-81.

Klann, R. C., \& Beuren, I. M. (2012). Gerenciamento de Resultados: análise comparativa de empresas brasileiras e inglesas antes e após a adoção das IFRSs. Anais Do Encontro Nacional Da Associação Nacional de PósGraduação E Pesquisa Em Administração, Rio de Janeiro, RJ, Brasil, 36, 1-18.

Landsman, W. R., Maydew, E. L., \& Thornock, J. R. (2012). The information content of annual earnings announcements and mandatory adoption of IFRS. Journal of Accounting and Economics, 53(1-2), 34-54.

Leuz, C. (2003). IAS versus U.S. GAAP: Information asymmetry-based evidence from Germany's new market. Journal of Accounting Research, 41(3), 445-472.

Leuz, C., \& Verrecchia, R. E. (2000). The Economic Consequences of Increased Disclosure. Journal of Accounting Research.

Li, S. (2010). Does Mandatory Adoption of International Financial Reporting Standards in the European Union Reduce the Cost of Equity Capital? The Accounting Review, 85, 607-636.

Niyama, J. K. (2010). Contabilidade Internacional. (Atlas, Ed) (2nd ed). São Paulo.

Niyama, J. K., \& Silva, C. A. T. (2013). Contabilidade Internacional. (Atlas, Ed.). São Paulo.

Nobes, C., \& Parker, R. (2010). Comparative International Accounting. (Pearson, Ed.) (11th ed.). London. 
Ohlson, J. A., \& Zhang, X.-J. (1998). Accrual Accounting and Equity Valuation. Journal of Accounting Research, 36(May), 85-111.

Olson, D., \& Mossman, C. (2003). Neural network forecasts of Canadian stock returns using accounting ratios. International Journal of Forecasting, 19(3), 453-465.

Ou, J. A., \& Penman, S. H. (1989). Financial statement analysis and the prediction of stock returns. Journal of Accounting and Economics, 11(4), 295-329.

Paananen, M., \& Lin, H. (2008). The Development of Accounting Quality of IAS and IFRS Over Time: The Case of Germany. Journal of Chemical Information and Modeling, 53(9), 1689-1699.

Perotti, P., \& Wagenhofer, A. (2014). Earnings Quality Measures and Excess Returns. Journal of Business Finance \& Accounting, 41(5-6), 545-571.

Pimentel, R. C., \& Aguiar, A. B. (2012). Persistence of quarterly earnings: an empirical investigation in Brazil. Brazilian Business Review, 9 (special edition BBR Conference), 38-54.

Psaros, J., \& Trotman, K. T. (2004). The Impact of the Type of Accounting Standards on Preparers' Judgments. Abacus, 40(1), 76-93.

Ribeiro, R. B., \& Da Silva, J. D. G. (2010). Estágio de Adequação das Informações Contábeis às Normas Internacionais de Contabilidade no Setor de Petróleo, Gás e Biocombustíveis. Revista de Educação E Pesquisa Em Contabilidade, 4(3), 70-93.

Samarasekera, N. (2013). Accounting quality of UK firms under IFRS. UWA Business School.

Santos, M. A. C., \& Cavalcante, P. R. N. (2014). O efeito da adoção dos IFRS sobre a relevância informacional do lucro contábil no Brasil. Revista Contabilidade \& Finanças, 25(66), 228-241.

Santos, L. P. G., Lima, G. A. S. F., Freitas, S. C., \& Lima, I. S. (2011). Efeito da Lei 11.638/07 sobre o conservadorismo condicional das empresas listadas BM\&FBOVESPA. Revista Contabilidade \& Finanças, 22(56), 174-188.

Sengupta, P. (1998). Corporate disclosure quality and the cost of debt. The Accounting Review, 73(4), 459.

Silhan, P. A. (2014). Income smoothing from a Census X-12 perspective. Advances in Accounting, 30(1), $106-115$.

Sousa, E., Sousa, A. F. de;, \& Demonier, G. B. (2016). Adoção das IFRS no Brasil: Efeitos no Conservadorismo Contábil. Revista de Educação E Pesquisa Em Contabilidade, 10(2), 136-147.

Tan, H., Wang, S., \& Welker, M. (2011). Analyst following and forecast accuracy after mandated IFRS adoptions. Journal of Accounting Research, 49(5), 1307-1357.

Tarca, A. (2004). International Convergence of Accounting Practices : Choosing between IAS and US GAAP. Journal of International Financial Management and Accounting, 15(1).

Tendeloo, B. V., \& Vanstraelen, A. (2005). Earnings Management under German GAAP versus IFRS. European Accounting Review, 14(1), 155-180.

Tucker, J. W., \& Zarowin, P. a. (2006). Does Income Smoothing Improve Earnings Informativeness ? The Accounting Review, 81(1), 251-270.

Yoon, S. (2007). Accounting Quality and International Convergence. PhD Proposal, 1. 